\title{
Volume of Distribution Steady State Observed by Bound Drug
}

National Cancer Institute

\section{Source}

National Cancer Institute. Volume of Distribution Steady State Observed by Bound Drug. NCI Thesaurus. Code C156574.

The volume of distribution at steady state based on the observed CLST for a substance administered by extravascular dosing, divided by the fraction of bound drug. 